\title{
Impact of Forming, Welding, and Electropolishing on Pitting and the Surface Finish of SRF Cavity Niobium
}

\author{
L. D. Cooley Member IEEE, D. Burk, C. Cooper, N. Dhanaraj, M. Foley, D. Ford, K Gould, D. Hicks, \\ R. Novitski, A. Romanenko, R. Schuessler, C. Thompson, and G. Wu
}

\begin{abstract}
A broad range of coupon electropolishing experiments are described to ascertain the mechanism(s) by which large defects are formed near superconducting radiofrequency (SRF) cavity welds. Cold-worked vs. annealed metal, the presence of a weld, and several variations of electropolishing (EP) parameters were considered. Pitting is strongly promoted by cold work and agitation of the EP solution. Welding also promotes pitting, but less so compared with the other factors above. Temperature increase during EP did not strongly affect glossiness or pitting, but the reduced viscosity made the electrolyte more susceptible to agitation. The experiments suggest that several factors that are rather benign alone are combined by the cavity forming, welding, and processing sequence to promote the formation of defects such as pits. Process changes to mitigate these risks are discussed.
\end{abstract}

Index Terms-Superconducting radio-frequency cavities, niobium, electropolishing, linear accelerator, linear collider

\section{INTRODUCTION}

$S_{c}^{u}$ UPERCONDUTING radio-frequency (SRF) cavities are the central components of advanced linear colliders for particle physics, nuclear physics, medical science, and other implementations of accelerated charged particles. Their distinct advantage over copper cavities is a much higher quality factor $Q$, by virtue of the very low surface resistance afforded by the superconducting state [1],[2]. The present technology is based on niobium, due to the favorable superconducting properties compared with other ductile superconducting materials at $2 \mathrm{~K}$ and $\sim 1 \mathrm{GHz}$. The cavity grade of niobium [3],[4] has high purity, good formability, is amenable to electron beam welding, and typically takes on a shiny, smooth surface finish after electropolishing.

The state of the art might best be represented by 9-cell 1.3 $\mathrm{GHz}$ cavities with the elliptical TESLA cell design. Optimization of this design is the focus of the International Linear Collider (ILC) project. Accelerating gradients above the ILC specification of $35 \mathrm{MV} \mathrm{m}^{-1}$ can now be attained by a sizeable fraction of cavities that have been given a standard

Manuscript received 3 August 2010. This work was supported under contract no. DE-AC02-07CH11359 with the United States Department of Energy.

All authors are with the SRF Materials Group, Technical Division, Fermi National Accelerator Laboratory. Contact: Lance Cooley, +1-630-840-6797, ldcooley@fnal.gov, P.O. Box 500, Mail Stop 316, Batavia IL 60510 U.S.A. electropolishing (EP) process, whether or not the cavities are supplied by multiple vendors or processed at different laboratories [5]-[7]. This is a significant milestone, given that production of the $\sim 20,000$ cavities needed for the ILC will require a tremendous industrial effort with high yield.

However, yield is far from optimum. A broad range of cavity performance results even though the same material, fabrication, and processing is followed by a single vendor or laboratory [5]-[7]. Just as large a fraction of cavities have sub-par performance, below $30 \mathrm{MV} \mathrm{m}^{-1}$, as the fraction which meets the ILC specification above when given the standard processing treatment. Additional electropolishing (EP), which adds cost and time, is sometimes, but not always, successful at improving performance. Further, the rate of occurrence of cavities that perform very poorly, barely reaching $20 \mathrm{MV} \mathrm{m}^{-1}$ after receiving a standard processing sequence, is not negligible [5]-[7]. What is worse is that some of these cavities do not respond at all to additional EP, which takes them out of the effective cavity inventory until some repair technique can be attempted.

Sub-par and, especially, poor cavities often exhibit quenches at particular locations, as revealed by varying the resonating mode to isolate bad cavity cells and by locating the quench with external thermometer arrays [2],[8]. When the quench location is subsequently examined by new cavity optical inspection tools [9], large pits, bumps, and other features are found [8],[10]. Typical sizes are 0.1 to $1.0 \mathrm{~mm}$ width and 10 to $100 \mu \mathrm{m}$ depth [10]. The mechanism by which the defects cause quench is presently being debated; whatever the mechanism is, what is clear is that preventing such defects from forming could significantly improve cavity yield.

The SRF Materials Group at Fermilab has undertaken a range of experiments to understand how large cavity defects form. The results of some of these experiments are reported in this article. No single origin of defects was found; instead, it appears that multiple effects combine to increase tendencies for defects to be formed. This means that process modifications in multiple areas may be required to prevent or reduce the likelihood of performance-limiting defects. We discuss how procedures might be changed.

\section{EXPERIMENT DESIGN AND PROCEDURES}

Optical inspection systems have provided many images of cavity welds over the past 2 years [10],[11]. These images 
show some common features, namely:

1) Defects are often located at the boundary between the molten zone of the weld and the solid recrystallized metal. Defects in this region often have a hemispherical shape and a depth to width ratio of approximately 1:10.

2) Defects also are often located at the edge of the heataffected zone (HAZ), between recrystallized metal and recovered or unaffected metal. Defects in this region often have a more faceted appearance, and grain boundaries are sometimes evident inside the defect.

Defects have been noted for both equator and iris welds. Typically the iris weld is performed as a partial penetration from the outside followed by a deeper penetration from the inside. The equator weld comes later in the sequence and is performed from the outside in a series of 3 passes: tack, seal, and full penetration. Beyond this, however, there is a range of variability due to art, the experience of the operator, tooling, the vacuum chamber, pre-weld preparations, the electron source, focusing and rastering of the beam, machining and alignment of the half cells, and so on. These variations are, evidently, not decisive to first order since cavities produced by all vendors exhibit the general defect features above.

In addition to welding, other factors should be considered. Niobium sheets are received in a 95\% recrystallized state and have grain size typically ranging from ASTM 4 to ASTM 6 (about the same size range as cavity defects) [3],[4]. When they are formed into half cells by stamping or hydroforming, moderate elongation occurs at the iris and both radial elongation and circumferential compression occurs at the equator. This cold work is generally not recovered prior to welding. Electropolishing is typically applied in two steps [12], a heavy EP where $\sim 150 \mu \mathrm{m}$ of material is removed, and a light EP where $\sim 25 \mu \mathrm{m}$ of materials is removed. This is routinely done in a horizontal tool in which $60 \%$ of the volume is filled with acid electrolyte and the remaining space is filled with a nitrogen cover gas flow to remove hydrogen. The cavity spins slowly at $\sim 1 \mathrm{rpm}$, drawing a meniscus of electrolyte along its inner surface. Between the heavy and light EP, a high-vacuum baking at $600-800{ }^{\circ} \mathrm{C}$ is applied to outgas hydrogen from the cavity. The light EP is followed by a final high-pressure rinsing with ultrapure water, after which the cavity is assembled with couplers in a clean room, evacuated, baked at $120^{\circ} \mathrm{C}$ under vacuum, and tested.

It is during this last test step that quench locations are first determined, and subsequently optical inspection is used to examine the quench locations. Thus, any of the steps above could contribute to the formation of cavity defects. However, there are no reports of new cavity defects being created by repeating steps after the $600-800{ }^{\circ} \mathrm{C}$ bake, so these can be excluded.

Based on the information above, the following mechanisms were considered as being most plausible for defect formation:

1) Pitting assisted by stored cold work.

2) Stress-induced pitting.

3) Etching defects due to pile-up of dislocations or interstitial atoms at the edge of the HAZ.

4) Coalescence of weld pores.

5) Vaporization of impurities due to poor cleaning.
6) Spitting, blow-through, arcing, and other phenomena associated with welding.

7) Agitation or thinning of the electrolyte next to the niobium surface, resulting in local chemical attack that is not limited by the fluorine ion gradient.

With these factors in mind, we chose to systematically explore the effects of electropolishing on a series of niobium coupons with the following experimental variables:

- Cold-worked vs. annealed Nb.

- Welded coupons vs. coupons with no weld.

- Welding by 3 different vendors.

- Electropolishing with still and agitated solutions.

- Electropolishing at $50{ }^{\circ} \mathrm{C}$, in addition to $30{ }^{\circ} \mathrm{C}$ as is typically done, to reduce viscosity and promote any deleterious trends.

We also keep other parameters constant, with characteristics as close to parameters used for cavity processing as possible. This includes:

- Metal thickness and machining preparations.

- Pre-weld preparation: Degreasing via ultrasonic cleaning with detergent followed by a 20 minute etch in 2:1:1 buffered chemical polishing (BCP) solution (98\% phosphoric acid, 99\% nitric acid, 48\% hydrofluoric acid) at $10{ }^{\circ} \mathrm{C}$ for $\sim 20 \mu \mathrm{m}$ material removal.

- Weld sequence and parameters: tack, seal, and full penetration toward the "RF side", using Fermilab specifications [13] for de-focus, raster, feed rate, etc.

- EP solution: 9:1 by volume $98 \%$ sulfuric acid and $48 \%$ hydrofluoric acid.

- Material removed during EP: 150 to $200 \mu \mathrm{m}$.

- Cathode material: 1100 series Al.

- EP parameters: $14.5 \mathrm{~V}$ (Al cathode to $\mathrm{Nb}$ anode), floating current ( $50 \mathrm{~mA} \mathrm{~cm}{ }^{-2}$ at $30{ }^{\circ} \mathrm{C}$ ), temperature control via water bath heat exchanger to $1^{\circ} \mathrm{C}$.

Annealed coupons were drawn from Fermilab cavity-grade stock [4], being cut from the same $2.8 \mathrm{~mm}$ thick sheets as were used to fabricate recent SRF cavities. Coupons were cut by wire electrical discharge machining (EDM) to $7.5 \mathrm{~cm}$ squares (no-weld samples) or into triangular pieces (weld samples) as shown in Fig. 1. The diagonal edge to be joined during welding was machined to $1.7 \mathrm{~mm}$ thickness $8.3 \mathrm{~mm}$ back from the diagonal, which is characteristic of TESLA cavity equator weld parameters [13].

Cold-worked coupons were made by rolling annealed sheet from $3.3 \mathrm{~mm}$ thickness to $1.7 \mathrm{~mm}$ thickness using a 2-high rolling mill at the Applied Superconductivity Center, National High Magnetic Field Laboratory, Florida State University. Approximately $10 \%$ thickness reduction was applied per pass, with pieces rotated by $90^{\circ}$ between passes. Weld samples were cut by EDM first into $6.2 \mathrm{~cm}$ squares and then separated along the diagonal as also shown in Fig. 1.

Pre-weld etching was carried out at Fermilab using the same equipment and facilities as used for other niobium welding preparations. Following degreasing and BCP etching, the pieces were rinsed repeatedly in ultrapure water, dried with clean nitrogen gas (boil-off from liquid), packaged in air-tight bags and shipped to the welder. The weld preparations were 
carried out no later than 48 hours before the welding took place.

\section{Annealed coupons Cold-worked coupons}

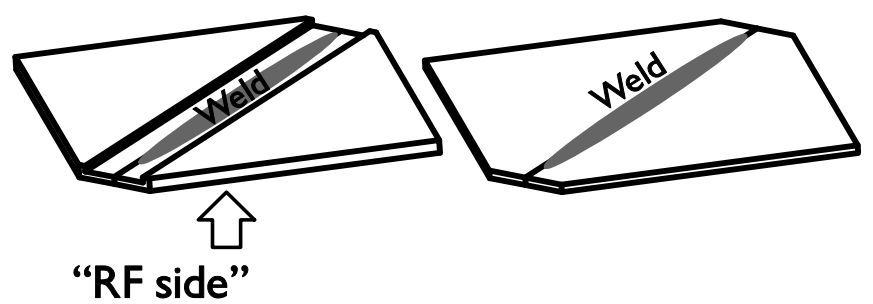

Fig. 1. Sketch of the coupon geometry and weld orientation. The electron beam trajectory was such that full penetration was made toward the side designated as the "RF side". For the annealed coupons, this side is opposite the machined surface, in analogy with cavities.

Coupon welding took place at Advanced Energy Systems, Inc. (Medford, NY), Pavac Industries (Richmond, BC), and at Sciaky Inc. (Chicago, IL). A Fermilab operator performed the welding at Sciaky. Vendors will not be identified later in this paper. Clamping and tooling to hold the pieces together and align the diagonals with the weld was left up to the vendor. Fig. 1 shows the coupons and the weld geometry.

Coupon electrochemistry took place at Fermilab using a bench-top system specifically designed and built for this purpose. A pneumatic diaphragm pump was used to circulate acid through a $2 \mathrm{~L}$ beaker where the polishing took place, drawing the acid through an aluminum heat exchanger coil submerged in a water bath and back to the beaker. Thermocouples measured the temperature in the acid bath and at the surface of the $\mathrm{Al}$ coil at the inlet and the outlet of the water bath. The temperature of the water bath was adjusted at the start of the process to maintain EP temperature within $1{ }^{\circ} \mathrm{C}$ over 4-5 hours. Typically this adjustment phase lasted during the first 30 minutes of an EP run. Fresh EP solution was mixed for all work reported here. The Al cathode and $\mathrm{Nb}$ anode were suspended in the electrolyte by $\mathrm{Nb}$ wires looped through holes above the bath, to which electrical connections were made. Voltage, current, temperature, and other data were recorded by a computer.

\section{COUPON ANALYSES}

\section{A. Metrics and Benchmarks}

The total amount of material removed was determined by measuring the mass before and after the EP run and noting the submerged area of the sample. These measurements were compared with those taken by a digital micrometer in a corner of the sample. The micrometer measurement routinely left scratches on the shiny sample surface, so this technique was used sparingly.

The coupons were visually inspected before and after every electropolishing run. In most cases, a silicone replica was also obtained to keep a record of the surface topography and to be used for surface scanning by profilometer without risk of damage to the samples (annealed niobium is very soft, and scratches by the profilometer stylus have been observed on other samples).
The quality of the electropolishing was determined by measurements of gloss and roughness. A gloss meter, which measures reflected light intensity from a calibrated source, was used to quantify the shininess of the surface in terms of "gloss units" GU. A high-quality electropolished stainless steel block (594 GU) and a black anodized aluminum block (94 GU) were provided as standards. Micro- and macroroughness were measured with a stylus profilometer using $1 \mu \mathrm{m}$ and $1 \mathrm{~mm}$ scans. Roughness is known to obey fractal behavior for SRF niobium [14], so peak and average roughness are not meaningful without the scan dimension. A clear correlation was found between both parameters and gloss, where the correlation for micro-roughness is shown in Fig. 2. Henceforth, therefore, we report only gloss measurements. Finally, area scans were also used to obtain topographies of defects.

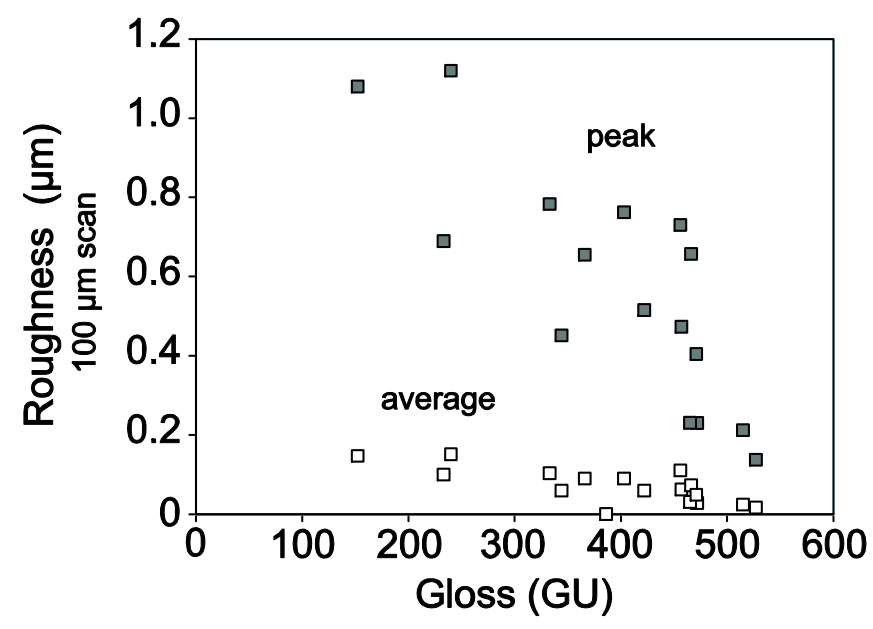

Fig. 2. Comparison of average and peak roughness as a function of gloss. The roughness data were acquired for a $100 \mu \mathrm{m}$ line scan.

A difficult issue is producing objective assessments of coupon surfaces that effectively capture features of most interest to SRF cavities. Closer examinations naturally yield more details, so efforts were made to benchmark examinations with standard procedures and reference samples. A flat bed scanner was used to obtain images of the coupons at $600 \mathrm{dpi}$ resolution. These images contained significant speckle and other contrast variations which might be interpreted as pits or other defects. When 600 dpi images were re-sampled to 72 dpi, the re-sampling effectively filtered out contrast variations except for only the largest features, as demonstrated in Fig. 3. For instance, a $500 \mu \mathrm{m}$ wide pit would produce $12 \times 12=144$ pixels of bright area in the original scan $(42 \mu \mathrm{m}$ per pixel) and $2 \times 2=4$ pixels of bright area in the re-sampled image, whereas a $100 \mu \mathrm{m}$ wide pit $(2 \times 2)$ would fall to the edge of bicubic re-sampling at $72 \mathrm{dpi}$ and produce little to no contrast.

A sample with a clearly located $500 \mu \mathrm{m}$ wide hemispherical defect was used as a reference to guide screening of coupon images. This is also shown in Fig. 3. Areas of high contrast were then analyzed with the profilometer to ensure that the contrast variation was not caused by debris, scratches, or other features. Since light is acquired at an angle offset from the illuminating filament, some diffusive scattering is required to 
produce a bright pixel. Thus, black regions represent mirrorlike gloss.
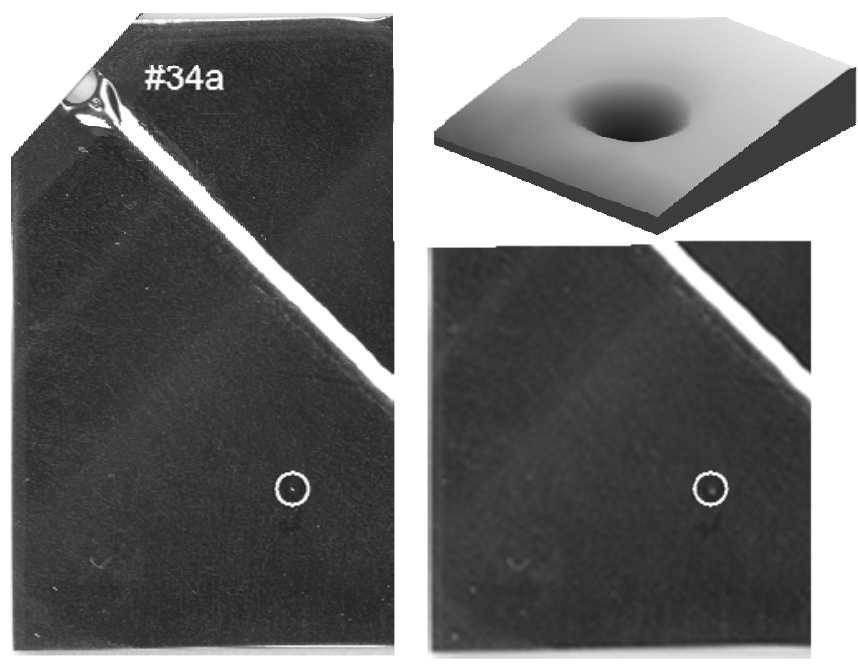

Fig. 3. Benchmark sample used as a reference for identification of pits. At left is shown a cropped image at actual size and 600 dpi as obtained from a flat-bed scanner. Black regions represent high gloss due to lack of diffusive scattering. A large pit is indicated by the circle. At lower right is the same image re-sampled to $72 \mathrm{dpi}$. Notice that significant contrast change remains inside the indicated area after the re-sampling. Inset at top right is a stylus profilometry scan $1 \mathrm{~mm} \times 1 \mathrm{~mm} \times 50 \mu \mathrm{m}$ high, showing the dimensions of the pit to be approximately $500 \mu \mathrm{m}$ across.

\section{B. Image Analyses}

Table 1 summarizes the analyses of a sub-set of the coupons studied chosen to illustrate the different trends in the scanned images. Clearly, chemical etching produced much higher gloss than for the as-received coupon. Also, annealed coupons could be electropolished to mirror-like finish with gloss almost as high as the stainless steel calibration standard. This validates the EP procedure.

Examples of an annealed weld coupon and a cold worked coupon are shown in Fig. 4. In both cases, gloss like that of the annealed EP coupons is indicated by the generally black appearance of the scans. Some pits were identified in the annealed weld coupons, as indicated in the figure. Coldworked coupons exhibited a starkly different appearance, as the example in Fig. 4 shows. Within the glossy regions, numerous speckles appear with sufficient contrast to pass the identification criterion discussed earlier. Closer inspection with profilometry of a couple of these regions, shown in Fig. 5, reveals that a mixture of many smaller pits accompanies the large pits. The number of small pits is at least an order of magnitude higher than for the large pits, suggesting that there are hundreds of small pits per square centimeter.

Examples of cold-worked welded coupons are shown in Fig. 6. In addition to features seen for cold-worked coupons, such as numerous pits across the entire area, and for welded annealed coupons, such as a few large pits located near the weld, new features were seen at the boundary of the weld bead. These features, indicated by the arrows in Fig. 6, appear as dark spots and have a characteristic size of 200 to $500 \mu \mathrm{m}$ and spacing of $\sim 1 \mathrm{~mm}$. Profilometry was difficult to obtain for these regions due to their proximity to the curved bead.
We speculate that these dark spots are bowl-shaped features with high reflectance at normal incidence.

TABLE 1 EXPERIMENTAL RESULTS

\begin{tabular}{|c|c|c|c|c|c|}
\hline $\begin{array}{c}\text { Sample } \\
\text { (welder) }\end{array}$ & $\begin{array}{c}\text { EP Temp. } \\
\left({ }^{\circ} \mathrm{C}\right)\end{array}$ & $\begin{array}{c}\text { EP time } \\
\text { (min) }\end{array}$ & $\begin{array}{c}\text { Nb loss } \\
(\mu \mathrm{m})\end{array}$ & $\begin{array}{l}\text { Gloss } \\
\text { (GU) }\end{array}$ & $\begin{array}{c}\text { Pits }^{\mathrm{a}} \text { per } \\
50 \mathrm{~cm}^{2} \\
\end{array}$ \\
\hline Before EP & -- & -- & -- & $\sim 30$ & -- \\
\hline \multicolumn{6}{|c|}{ Annealed, no weld } \\
\hline EP \#4 & 31 & 120 & 160 & 456 & 0 \\
\hline EP \#5 & 30 & 155 & 176 & 527 & 0 \\
\hline EP \#6 & 56 & 103 & 253 & 472 & 3 \\
\hline \multicolumn{6}{|c|}{ Cold-worked, no weld } \\
\hline CW \#3 & 30 & 298 & 190 & 422 & $>50$ \\
\hline CW \#5 & 52 & 110 & 219 & 398 & $>50$ \\
\hline \multicolumn{6}{|c|}{ Annealed, welded } \\
\hline$\# 1$ (C) & 30 & 332 & 208 & 457 & 9 \\
\hline \#3 (C) & 24 & 279 & 211 & 403 & 9 \\
\hline$\# 25$ (A) & 30 & 301 & 205 & 381 & 2 \\
\hline$\# 29$ (A) & 50 & 123 & 198 & 432 & 8 \\
\hline$\# 17$ (B) & 30 & 301 & 135 & 424 & 2 \\
\hline \#18a (B) & $50^{\mathrm{b}}$ & 123 & 77 & 172 & -- \\
\hline \#21b (B) & $51^{\mathrm{c}}$ & 335 & 240 & 134 & $>10$ \\
\hline \multicolumn{6}{|c|}{ Cold-worked, welded } \\
\hline \#34a (C) & 50 & 300 & 206 & 478 & $>10$ \\
\hline \#36 (C) & 52 & 145 & 137 & 449 & $>50$ \\
\hline \#35a (C) & $58^{\mathrm{d}}$ & 130 & 92 & 153 & -- \\
\hline
\end{tabular}

${ }^{\mathrm{a}}$ Pits must pass the contrast criteria, i.e. they are $\sim 500 \mu \mathrm{m}$ in size

${ }^{\mathrm{b}}$ High flow, small cathode, high temperature.

${ }^{\mathrm{c}}$ Coupon was cyclically dipped into the warm EP solution.

${ }^{\mathrm{d}}$ Ultrasonic agitation was applied during EP at high temperature.
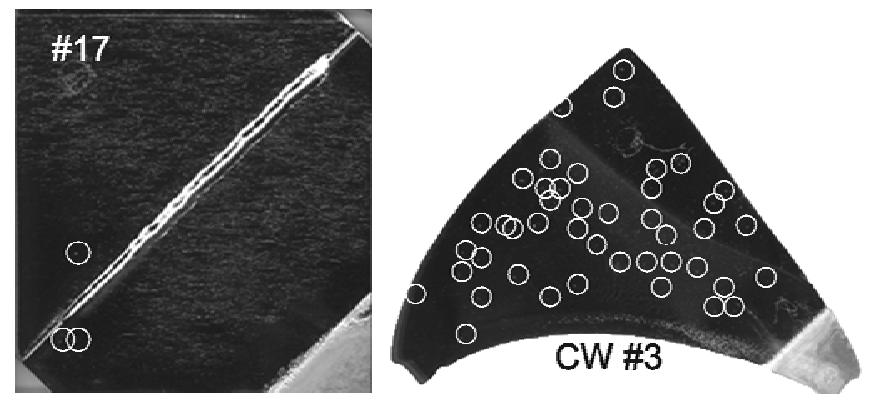

Fig. 4. At left is an annealed welded coupon from welder B. A mirror-like finish comparable to annealed coupons with no weld is seen. However, a few large pits are also located near the weld as indicated by the circles. At right is a cold-worked coupon, with many pits indicated by the circles. The distorted triangular shape is due to the deformation of rolling, where the starting piece was a triangular half-coupon. Images are shown at $220 \mathrm{dpi}$.
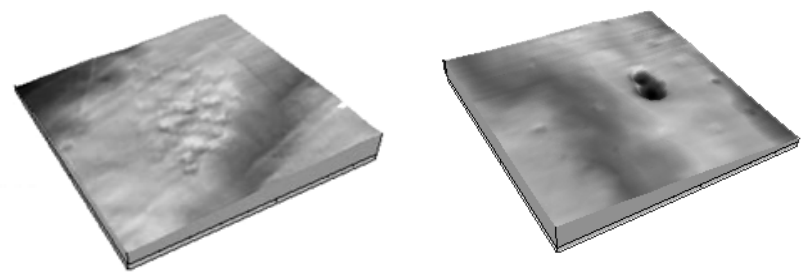

Fig. 5. Profilometer scans of different regions of CW \#3. At left is a region 1 $\mathrm{mm}$ x $1 \mathrm{~mm}$ x $25 \mu \mathrm{m}$ high, while at right is a region $1 \mathrm{~mm}$ x $1 \mathrm{~mm}$ x $50 \mu \mathrm{m}$ high. Notice the mixture of large and small pits in the right profile. 
Finally, a set of coupons processed with various forms of agitation given to the electrolyte is shown in Fig. 7. The implications of various agitation techniques will be discussed shortly. It is important to note that, in all of these examples, high temperature was important for exacerbating the effects of the agitation; experiments at $30{ }^{\circ} \mathrm{C}$ yielded inconsistent results. Annealed weld coupon \#18a was processed with a small cathode, $1 / 20$ the area of the niobium, which allowed much higher flow of electrolyte past the coupon surface. Grain contrast in this image is suggestive of typical etching processes used in metallography. The HAZ is difficult to determine due to the large grain size of this annealed metal. Ultrasonic agitation was applied during the electropolishing of cold-worked weld coupon \#35a, which produced a similar effect as for coupon \#18a but over much shorter time. The HAZ is clearly visible due to the fine grain structure of the cold-worked metal. Annealed coupon \#21b was cyclically dipped into the electrolyte with a period of $\sim 10 \mathrm{~s}$. Again, grain contrast is seen in the central region where a meniscus coated the sample. The region at bottom left remained in the EP solution and took on a much better gloss, free from visible grain boundaries. Many $(>10)$ large pits were identified in the central meniscus region of \#21b and located in the HAZ.

It is very important to note that the meniscus region of \#21b has the closest resemblance to the grain SRF cavity images.
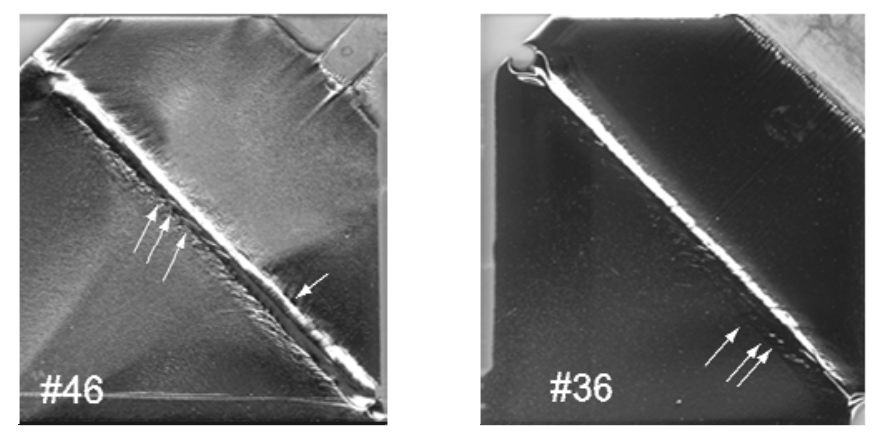

Fig. 6. Cold-worked welded coupons. The arrows indicate new features not seen for either cold-worked coupons or annealed weld coupons.

\section{DISCUSSION}

\section{A. Agitation Promotes Etching}

Clear grain contrast replaced a glossy surface finish when the electrolyte was agitated. Agitation produces mixing at the niobium surface, which allows free fluorine to be re-supplied to the locations where it is consumed by chemical reactions which convert niobium pentoxide into soluble fluoride salts. Under normal EP conditions, free fluorine must diffuse through highly viscous sulfuric acid, which establishes a diffusion-limited fluorine gradient of several tens of micrometers in thickness [15]. The lack of available fluorine near the oxidized surface, and the abundance of fluorine above the surface, produces faster etching of peaks and promotes reduction of roughness, i.e leveling. When fluorine is readily available at the niobium surface, other factors, including the etch rates of various crystallographic directions and the availability of dislocations and other defects to assist oxidation, determine the surface finish.
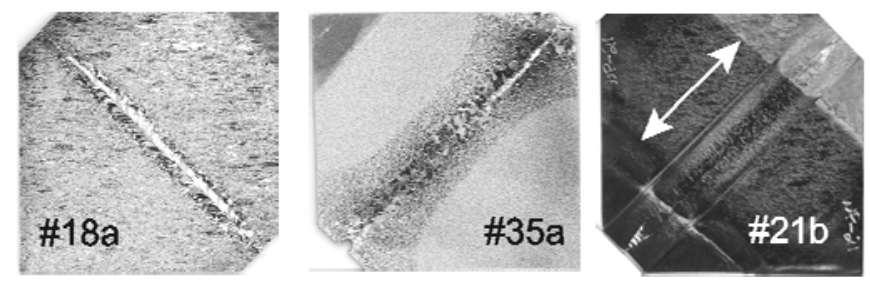

Fig. 7. Weld coupons polished with some form of agitation of the electrolyte. At left, \#18a was processed with a higher flow of electrolyte past the sample. In the center, \#35a was processed with ultrasonic agitation applied during EP. At right, \#21b was processed by cyclically dipping the coupon into the electrolyte, where the arrows indicate the region of the coupon coated by a meniscus. All of these coupons previously were polished to high gloss by standard EP. Notice the grain contras revealed by these procedures, which is akin to grain contrast revealed by BCP and other etching techniques.

The agitation was effective for revealing the HAZ for coldworked specimens. This is due to the substantial difference in grain size between the recrystallized portion of the HAZ and the unaffected metal. Agitation apparently exacerbates pitting, as suggested by the large pits seen on samples \#21b and \#35a. This might be expected when local chemical attack can be accelerated by supplying fluorine to the coupon surface.

\section{B. Cold-Working Strongly Promotes Pitting}

The difference in defect numbers between annealed and cold-worked coupons is rather astonishing. Cold working clearly promotes the formation of defects at many scales, not just the large scales comparable to the $500 \mu \mathrm{m}$ reference defect. Profilometry scans such as those shown in Fig. 5 clearly detail much greater activity at scales less than $100 \mu \mathrm{m}$. Further study by electron microscopy, laser confocal scanning microscopy, and atomic force microscopy is underway to understand the fine detail. In particular, the role of dislocations in assisting etching processes is well known [16], and recent studies [17] have revisited this topic for cavitygrade niobium.

What is also interesting is that cold-worked coupons take on as high a gloss as annealed coupons, despite the numerous defects. We speculate that cold working mixes textures, which reduces the availability of large areas with common crystallographic orientation that could be etched rapidly.

\section{High EP Temperature}

Generally, all annealed samples took on a high gloss with few pits. The observed changes for samples with EP at $50^{\circ} \mathrm{C}$ were not significant compared with samples polished at $30^{\circ} \mathrm{C}$. This suggests that, by itself, electropolishing at higher temperatures does not instigate the formation of defects. However, increase of temperature had a significant effect on the viscosity of the electrolyte, which played a contributing role in the other effects noted above. In particular, we used this fact to more easily agitate the electrolyte. During cavity processing, EP temperatures can reach 45 to $50{ }^{\circ} \mathrm{C}$ at the cavity walls despite the temperature of the incoming electrolyte being $30{ }^{\circ} \mathrm{C}$, mostly due to the exothermic oxidation reaction of niobium [15]. When coupled with cavity rotation, a low-viscosity meniscus probably coats the cavity surface as it is drawn up out of the electrolyte. This is probably why sample \#21b produces a similar microstructure 
as seen in cavity optical images.

\section{Welding Promotes Pitting}

Despite the high gloss and tendency of annealed samples to be defect-free, welding promoted a few large pits to form in these samples. The location of the pits is consistent with the zone where thermal stress could accumulate. Welding by itself did not play as strong a role as cold work.

\section{E. Combined Factors}

A main observation of this work is that many factors can be relatively benign by themselves, but can lead to significant rates of defect formation when they are combined. Combination of cold work and welding in particular led to the formation of new types of contrast variations near the weld bead, as indicated in Fig. 6. Closer inspection by light microscopy showed that these dark features were bowl-shaped dimples and gaps. As explained earlier, profilometry was not possible due to the strong surface contour of the weld bead.

\section{CONCLUSIONS AND IMPLICATIONS}

The coupon experiments described herein suggest that the present cavity fabrication procedure may link together several factors that produces a tendency to form weld defects. Namely, cold work put into the half cells during the forming process is not removed by a separate annealing step prior to welding, which adds a strong tendency for defect formation. Welding also adds a mild tendency for defect formation in the HAZ, and when combined with cold work there is an additional tendency to produce curious defects at the weld bead edge. These defects were not seen for any other experiment conditions. Rotation of the cavity during horizontal EP, combined with heating at the cavity wall, promotes agitation of the electrolyte, which the experiments here show will promote etching over leveling. Etching attack on these pre-conditions left by forming and welding were shown to strongly drive defect formation.

The results hold the following implications for changing the present cavity process:

1) Cavities should be annealed prior to EP.

2) Half cells should be annealed prior to welding.

3) The cavity wall should be cooled to maintain high electrolyte viscosity. High viscosity may mitigate the risks of agitation below.

4) If possible, rotation or other agitation should be avoided. Other meniscus films, such as those around bubbles in the electrolyte, present similar circumstances.

\section{ACKNOWLEDGMENT}

We are grateful to Bill Starch for assistance in preparing cold worked samples at Florida State. We also thank John Rathke at AES and Ralf Edinger at Pavac for coordinating welding of the coupons.

\section{REFERENCES}

[1] H. Padamsee, RF Superconductivity, Weinheim, Germany: Wiley-VCH, 2009.

[2] H. Padamsee, J. Knobloch, and T. Hays, RF Superconductivity for Accelerators, $2^{\text {nd }}$ ed., Weinheim, Germany: Wiley-VCH, 2008.

[3] Standard Specification for Niobium and Niobium Alloy Strip, Sheet, and Plate, ASTM Standard B393-05.

[4] Technical Specification for High RRR Grade Niobium Sheet and Rod for use in Superconducting Cavities, Fermilab specification 5500-ES371037-A, 2007.

[5] C. Ginsburg, "ILC Americas Regional Team 9-cell S0 Cavities", maintained online at http://tdserver1.fnal.gov/project/ILC/S0/S0_coord. html .

[6] R. L. Geng, "Overview of high gradient SRF R\&D for ILC cavities at Jefferson Lab”, Proc. SRF 2009, Berlin, Germany, 2009, p. 213, https://accelconf.web.cern.ch/accelconf/SRF2009/papers/tuppo015.pdf .

[7] D. Reshke, L. Lilje, and H. Weise, "Analysis of RF results of recent nine-cell cavities at DESY”, Proc. SRF 2009, Berlin, Germany, 2009, p. 342, https://accelconf.web.cern.ch/accelconf/SRF2009/papers/tuppo051. pdf .

[8] M. S, Champion, L. D. Cooley, C. M. Ginsburg, D. A. Sergatskov, R. L. Geng, H. Hayano, Y. Iwashita, and Y. Tajima, "Quench-limited SRF cavities: Failure at the heat-affected zone”, IEEE Trans. Appl. Supercond., vol. 19, pp. 1384-1386, 2009.

[9] Y. Iwashita, Y. Tajima, and H. Hayano, "Development of high resolution camera for observations of superconducting cavities", Phys. Rev. ST Accel. Beams, vol. 11, 093501 (6 pp.), 2008.

[10] K. Watanabe, "Review of optical inspection methods and results", Proc. SRF 2009, Berlin, Germany, 2009, p. 123, https://accelconf.web.cern.ch/ accelconf/SRF2009/papers/tuobau01.pdf .

[11] C. Ginsburg, Fermilab, private communication, draft WG 1 Report to the Tesla Technology Collaboration, April 2010.

[12] TESLA Technology Collaboration, "Final surface preparation for superconducting cavities”, TTC-Report 2008-05, available at http:// flash.desy.de/reports_publications/tesla_reports/tesla_reports_2008/ .

[13] D. Proch, DESY Specification of Welded $1.3 \mathrm{GHz}$ Superconducting Resonators for TTF/VUV-FEL, MHF-SL 09-2005.

[14] H. Tian, W. Sommer, O. Trofimova, C. E. Reece, and L. Zhao, "Analysis of the topographic transformation of niobium surfaces under controlled EP conditions”, Proc. SRF 2009, Berlin, Germany, 2009, p. 422, https://accelconf.web.cern.ch/accelconf/SRF2009/papers/tuppo081. pdf.

[15] H. Tian, S.G. Corcoran, C. E. Reece, and M. J. Kelley, "The mechanism of electropolishing of niobium in hydrofluoric-sulfuric acid electrolyte", J. Electrochem. Soc., vol. 155, pp. D563-D568, 2008.

[16] P.R. Evans, "Dislocation Etch Pit Studies in Annealed and Deformed Polycrystalline Nb”, J. Less Common Metals, vol. 6, pp. 253-265, 1964.

[17] X. Zhao, G. Ciovati, C. E. Reece, and A. T. Wu, "Surface topography of "hotspot" regions from a single cell SRF cavity", Proc. PAC09, Vancouver BC, 2009, paper WE5pfp062, http://trshare.triumf.ca/ pac09proc/Proceedings/papers/we5pfp062.pdf. 